\title{
IMAGEN CORPORAL EN CENTROS FITNESS UN PROBLEMA A ABORDAR
}

Julia García Martínez, Ángela Sierra Robles, Eduardo Fernández-Ozcorta

\section{Email: julia.garmar@gmail.com}

\section{RESUMEN:}

Recientemente, en la sociedad, se ha comenzado a enfatizar sobre la belleza física (Eagly, Ashmore, Makhijani, y Longo, 1991), convirtiéndose en una obsesión por la búsqueda de la aptitud física a través del movimiento, centrándose en preocupaciones estéticas por encima de la salud (Davisy Cowles, 1991), especialmente en adultos. En este caso, se puede observar que la práctica de actividad física puede llegar a ser un instrumento para conseguir un fin estético, obviando tanto la salud física como la psicológica, siendo éste último, el motivo de dicha propuesta de estudio.

Este hecho está ampliamente constatado en diferentes contextos de uso de la actividad física de forma aislada, sin embargo, no se ha estudiado este fenómeno en la amplia gama de actividades que pueden desarrollarse dentro de un centro deportivo, ni las diferencias entre cada una de ellas.

Los resultados de esta propuesta de estudio pueden aportar estrategias para que la práctica de actividad física en centros fitness no suponga un perjuicio psicológico mayor que los beneficios físicos que obtengan los usuarios de dichos centros.

PALABRAS CLAVE: Imagen corporal; centros fitness; actividad física. 


\section{INTRODUCCIÓN}

La imagen corporal ha sido definida por diferentes autores. Cash, en 1989, la define como la exactitud con la que las personas perciben el tamaño de su cuerpo y de los pensamientos y sentimientos asociados con la opinión del individuo sobre su propio cuerpo. Más tarde, Thompson, Heinberg, Altabe y Tantleff-Dunn (1999) la definen como la representación interna que tiene una persona sobre su apariencia exterior. Tradicionalmente, la imagen corporal ha sido entendida como un constructo multidimensional compuesto por dos componentes independientes, percepción y actitud (Cash, 1989; Rowe, 1999), pero posteriormente, en el año 2000, Cash explica que la imagen corporal está compuesta por cuatro dimensiones: percepciones, pensamientos, sentimientos y comportamientos, no sólo de la apariencia física, sino también la competencia del cuerpo, su integridad biológica y la presencia de salud o enfermedad.

Uno de los constructos con los que se relaciona potencialmente es con la insatisfacción corporal. Ésta última es definida como aquellas creencias y sentimientos negativos que uno tiene acerca de la forma y el peso de su cuerpo (Garner, 2002). La relación que se presenta entre ambas es que cuanto mayor es la diferencia entre la imagen corporal ideal y la real, mayores son los niveles de insatisfacción de la imagen corporal (Lewis y Rosenblum, 1999).

Además, la imagen corporal se correlaciona con diferentes teorías (tabla1) que manifiestan las repercusiones psico-sociales que pueden variar según el estado de la imagen corporal de la persona.

\begin{tabular}{|c|c|}
\hline Teorías & Concepto \\
\hline $\begin{array}{l}\text { Auto-presentación } \\
\text { (Leary, 1954) }\end{array}$ & $\begin{array}{l}\text { Se refiere a la auto-presentación como el proceso de seguimiento y } \\
\text { control de cómo se es percibido y evaluado por los demás para } \\
\text { crear una buena impresión, lo cual, está fuertemente influenciado } \\
\text { por los ideales culturales del cuerpo, llegando a creer que deben } \\
\text { presentar un cuerpo delgado (Krane, Stiles-Shipley, Waldron, y } \\
\text { Michalenok, 2001). }\end{array}$ \\
\hline $\begin{array}{l}\text { Comparación social } \\
\text { (Festinger, 1954) }\end{array}$ & $\begin{array}{l}\text { Explica que la gente experimenta un deseo innato de } \\
\text { autoevaluarse, mediante la comparación de sí mismos con otras } \\
\text { personas que tienen los mismos atributos, como raza, edad, } \\
\text { apariencia, género... (Salovey y Rodin, 1984), siguiendo normas } \\
\text { culturales (Franzoi y Klaiber, 2007). }\end{array}$ \\
\hline $\begin{array}{l}\text { Sociocultural } \\
\text { (Hausenblas y Fallon, } \\
\text { 2006) }\end{array}$ & $\begin{array}{l}\text { Hace referencia a que la presión social, como medios de } \\
\text { comunicación, amigos, familia..., es la razón por la que la gente } \\
\text { quiere cumplir con un físico ideal difícil de cumplir sin seguir una } \\
\text { dieta, un programa de actividad física o ambos (Thompson, } \\
\text { Heinberg, Altabe y Tantleff-Dunn, 1999). }\end{array}$ \\
\hline $\begin{array}{l}\text { Ansiedad física social } \\
\text { (Leary, 1992) }\end{array}$ & $\begin{array}{l}\text { Ansiedad física social es la preocupación de la persona por cómo } \\
\text { los demás perciben su cuerpo. }\end{array}$ \\
\hline $\begin{array}{l}\text { teoría de la objetivación } \\
\text { (Fredrickson y Roberts, } \\
\text { 1997) }\end{array}$ & $\begin{array}{l}\text { Postula que si una persona observa su cuerpo en función de la } \\
\text { forma en que otros lo perciben, internaliza el exterior sexual y es } \\
\text { probable que perciba su cuerpo como un objeto }\end{array}$ \\
\hline
\end{tabular}

Tabla 1.- Resumen de las teorías asociadas al estudio de la imagen corporal. 
Actualmente, y desde la década de 1930, se considera que el atractivo sexual femenino depende de la delgadez (Garner, Garfinkel, Schwartz, yThompson, 1980). En este sentido, Koyuncu, Tok, Canpolat y Catikkas (2010) argumentan que los medios de comunicación y otras instituciones sociales nos muestran una imagen corporal ideal poco realista, a través de estrellas de cine y televisión, deportistas o modelos, cuya imagen es excesivamente delgada en comparación con los ideales del pasado, convirtiéndose en un estándar casi imposible de alcanzar para la mayoría de las personas, que puede influir de manera negativa en la forma en que las personas perciben sus cuerpos, pues tienden a compararlos con los de modelos profesionales. Por tanto, las mujeres centran su atención en los aspectos relacionados con el peso y el atractivo sexual (Franzoi y Klaiber, 2007), y los hombres en el aumento de la masa muscular (Thompson, Heinberg, Altabe, y Tantleff-Dun, 1999). En consecuencia, los mensajes de los medios de comunicación son responsables, en parte, de la insatisfacción con la imagen corporal. Van den Buick (2000) estudió que ver la televisión se correlaciona con el ideal de imagen corporal, y Harrison y Cantor (1997) constataron que las horas dedicadas a ver la televisión y a leer revistas de moda predijeron la insatisfacción con la imagen corporal, especialmente entre adolescentes y adultos jóvenes. Además, recientemente, se ha documentado que las revistas de fitness también exponen el mismo ideal de belleza, incluso con un mayor grado de desnudez (Wasylkiw, Emms, Meuse, y Poirier, 2009).

El trastorno de la imagen corporal causa estados afectivos negativos, debido a la teoría de auto-presentación (Leary, 1992), la teoría de la comparación social (Festinger, 1992) y la teoría sociocultural (Hausenblas y Fallon, 2006). Estas comparaciones pueden producir mayores niveles de ansiedad relacionados con el cuerpo (Sabiston, Crocker, y Chandler-Munroe, 2005).

Allgood-Merten, Lewinsohn y Hops (1990) argumentan que la imagen corporal es un aspecto importante de la autoestima, por tanto, la insatisfacción con la imagen corporal se asocia con bajos niveles de autoestima (Koff, Rierdan, y Stubbs, 1990; Bas, Asci, Karabudak, y Kiziltan, 2004). Según Johnson, Steinberg y Lewis (1988), cada vez más la delgadez se asocia con el logro personal, autocontrol, autonomía y el éxito, y por el contrario, la obesidad puede conducir a la discriminación social y a la baja autoestima. Así, la búsqueda de delgadez es el objetivo propuesto para obtener las respuestas sociales favorables, permitiendo aumentar la autoestima.

La discrepancia entre la imagen corporal que se desea y la que realmente se tiene, también pueden conducir a la ansiedad física social, En este sentido, existe una relación entre un mayor porcentaje de grasa corporal y un mayor índice de masa corporal (Hausenblas y Fallon, 2002), que a su vez se correlaciona con mayores niveles de ansiedad física social (Eklund y Crawford, 1994; Hart, Leary y Rejeski, 1989), con niveles inferiores de autoestima y con mayor insatisfacción con la imagen corporal tanto en hombres (Russell, 2002) como mujeres (Martin, Engels, Wirth y Smith 1997), siendo estas últimas las que tienen un mayor riesgo para tener insatisfacción corporal (Elgin y Pritchard, 2006; Feingold y Mazzella, 1998). Por lo tanto, la mayoría de los estudios se centran en la población femenina. Éstas se sienten con sobrepeso y quieren perder peso y buscar la delgadez (Thompson, Heinberg, Altabe y Tantleff-Dun, 1999). Sin embargo, gran parte de los hombres con negativa imagen corporal quieren aumentar su peso ganando masa muscular (McCabe y Ricciardelli, 2004), siendo en su mayoría hombres jóvenes, mientras que por norma 
general, los hombres mayores son más propensos a querer perder peso (Davis y Cowles, 1991), al igual que los homosexuales (McCreary, Hildebrandt, Heinberg, Boroughs y Thompson, 2007).

Esta diferencia entre sexos se debe, según Franzoi (1995), a la teoría de autoobjetivación, explicando que los hombres están socializados para juzgar sus organismos en términos de rendimiento, conceptualizando sus cuerpos como medio para alcanzarlo, mientras que las mujeres están socializadas para juzgar sus cuerpos en términos de apariencia, conceptualizando sus cuerpos como objeto, debido a la exposición a múltiples formas de objetivación del cuerpo (como una mirada sexual) (Prichard y Tiggermann, 2005). Un ejemplo claro con carácter deportivo de la repetición a lo largo del tiempo de dichas exposiciones, son las bailarinas o las gimnastas, ya que la apariencia de su cuerpo juega un papel importante en el rendimiento.

En el ámbito del deporte y de la actividad física, el estudio de la imagen corporal y de otros conceptos asociados a ésta cobra importancia, pues la participación en actividades deportivas tiene una influencia notable sobre éstos (Koyuncu et al., 2010).

Si analizamos la relación de la ansiedad física social con el ejercicio, encontramos que las personas sedentarias experimentan mayores niveles de ansiedad física social que las personas activas (Atalay y Gençöz, 2008). Sin embargo, Asçı, Tüzün y Koca, (2006) sugieren que las personas con elevados niveles de ansiedad física social son más propensas a involucrarse en un programa de ejercicio que aquellas con bajos niveles, pero a su vez, se encuentran más incómodos a la hora de la práctica física, pues sienten temor a ser vistos por los demás, por tanto, se vuelven más conscientes de su apariencia física, aumentando su ansiedad física social, por lo que tienden a reducir sus niveles de actividad física (Lantz, Hardy, y Ainsworth, 1997), llegando a estar más insatisfechos con su imagen corporal, y por ende, aumenta su ansiedad física social. En resumen, se genera un círculo vicioso que inhibe la mejora de la imagen corporal (Atalay y Gençöz, 2008). Aunque cabe destacar que, independientemente del nivel de ansiedad física social, ésta disminuye con el ejercicio (Atala, y Gençöz, 2008).

Davis, Kennedy, Ravelski y Dionne (1994) afirman que el ejercicio físico puede promover la insatisfacción con la imagen corporal por dos razones, por apariencia física o por eficacia física de la actividad realizada, pero existe controversia en cuanto a si las personas físicamente activas tienen una imagen corporal más positiva que las sedentarias (Ahmed, Hilton, y Pituch, 2002; Heinberg, 1995; Koff, y Bauman, 1997) o al contrario (Tiggemann y Williamson, 2000), pues según Greenleaf, McGreer, y Parham (2006), las personas que realizan ejercicio con frecuencia tienen generalmente una imagen corporal ideal difícil de alcanzar, y son más conscientes de su propia imagen. Tiggemann y Williamson (2000) explican que esta disyuntiva se genera por los motivos para la práctica. Las personas que practican ejercicio para perder peso o mejorar su atractivo tienen una elevada insatisfacción con su imagen corporal y una baja autoestima, son generalmente mujeres jóvenes y además no mejoran con la práctica (Strelan, Mehaffey, y Tiggemann ,2003). Mientras que las personas que practican actividad física por salud y por disfrute, poseen una satisfacción con su cuerpo elevada y suelen ser hombres y mujeres maduros. Esta diferenciación se produce porque las mujeres jóvenes están sometidas a más mensajes de los medios de comunicación que se dirigen específicamente a ellas, 
generando un ideal de belleza tan excesivamente delgado, que es imposible de alcanzar por mucho ejercicio que hagan. Lo único que consiguen es llegar a la decepción (Tiggemann y Williamson, 2000).

Pero los individuos no siempre pueden realizan ejercicio por la misma razón, el seguimiento de sus motivaciones día a día ofrece más información acerca de los efectos de las diferentes motivaciones para la práctica en un mismo individuo (LePage y Crowtheruna, 2010).

Se hace necesario estudiar el tipo de actividad que se relaciona con mejores percepciones de la imagen corporal, en este sentido, Prichard y Tiggemann (2008) demuestran que el tiempo dedicado a actividades de tipo aeróbico se relaciona con insatisfacción de la imagen corporal, sin embargo, no se encuentra relación con actividades al aire libre o entrenamiento con cargas e individuales. A pesar de todo, independientemente del nivel de insatisfacción corporal, el ejercicio es una experiencia positiva para las personas que hacen ejercicio con frecuencia (LePage y Crowtheruna, 2010).

Además, recientemente se ha documentado que hacer ejercicio regularmente sirve como protector contra la insatisfacción corporal (Duncan, Al-Nakeeb, Nevill y Jones, 2004), y la práctica de actividad física, ya sea aeróbica o anaeróbica, es un medio eficaz para la mejora de la imagen corporal (Henry, Anshel y Michael, 2006; Campbell y Hausenblas, 2009), de la ansiedad física social (Atalay y Gençöz, 2008) y de la autoestima (Maltby y Day, 2001), en lugar de la utilización de terapia cognitiva conductual, que ha sido utilizada como tratamiento tradicional (Cash, 1995), y además, en comparación con otras intervenciones, el ejercicio supone un bajo coste económico, es socialmente aceptable y no tiene efectos secundarios negativos (Campbell, y Hausenblas, 2009).

En cuanto a qué tipo de actividad es más adecuada, varía en función de cada persona (Ackard, Brehm, y Steffen, 2002; Calogero y Pedrotty 2007). Koff y Bauman (1997) concluyeron en su estudio que las mujeres que participaron en clases de step, en entrenamiento de fuerza o carrera continua mejoraron significativamente la imagen corporal, y por otro lado, se ha demostrado que en poblaciones adultas de diferente edad y género, mediante un entrenamiento de fuerza, se redujo la insatisfacción con la imagen corporal (Ahmed, Hilton y Pituch, 2002; Tucker y Maxwell ,1992) y la ansiedad física social (Williams y Cash, 2001) y se mejoraron los sentimientos de bienestar (Tucker y Maxwell ,1992). No obstante, Henry, Anshel y Michael (2006) demuestran que el entrenamiento de fuerza unido al aeróbico produce mejoras, significativamente superiores, en la imagen corporal que el entrenamiento aeróbico únicamente, debido a que también se mejora la aptitud física, y ésta se relaciona con mejoras en la imagen corporal (Skrinar, et al., 1986; Tucker y Maxwell, 1992; Koff \& Bauman, 1997). Por último, según Daubenmier (2005) las mujeres que practican yoga tienen mayor satisfacción corporal que las que realizan actividades aeróbicas, ya que esta modalidad está basada en la unificación de la mente y el cuerpo, aumentando la conciencia del cuerpo interior (Prichard y Tiggemann, 2008), aunque no existen más estudios que contrasten estas conclusiones. En cuanto a la frecuencia semanal de entrenamiento, Reboussin et al (2000) confirman que conforme ésta aumenta mejora la satisfacción corporal, sin embargo, en contraposición a esta idea, Prichard y Tiggemann $(2005 ; 2008)$ afirman lo contrario. Este dilema se debe a que no es necesariamente la cantidad o la frecuencia de ejercicio el factor importante, sino los 
conocimientos que las personas tienen hacia el ejercicio (Mond, Hay, Rodgers y Owen, 2006).Y por último, con respecto a la intensidad, ejercicios de intensidad moderada a extenuante obtienen mejoras mayores en la imagen corporal que intensidad leve, ya que dicho intervalo de intensidad es el que se requiere para recibir los beneficios relacionados con la salud de ejercicio (Hausenblas y Fallon, 2006). Las mejoras explicadas sobre la satisfacción de la imagen corporal pueden deberse a que se produce una sensación de que el cuerpo está mejorando a través del ejercicio, produciendo cambios como el peso corporal, musculatura, competencia física y percepción de salud (Campbell y Hausenblas, 2009).

En resumen, podemos afirmar, basándonos en estos estudios, que para obtener mayores mejoras en la percepción de la imagen corporal, se deben realizar tanto ejercicio de tipo aeróbico como anaeróbico, debido a que ambos son necesarios para alcanzar un buen estado físico general y conseguir un cuerpo que más se asemeje al ideal, ya que el ejercicio aeróbico es necesario para la pérdida de grasa o para prevenir el aumento de ésta, mientras que el anaeróbico desarrolla la masa muscular (Hausenblas y Fallon, 2006). Utilizando, al menos, una intensidad moderada.

Además, cabe destacar que las mayores mejoras en la imagen corporal se producen en mujeres que en hombres (Campbell y Hausenblas, 2009), porque las mujeres son más propensas a sufrir trastornos de la imagen corporal (APA, 1994). Con respecto a la edad, la imagen corporal mejora sea cual sea la edad (Garner, 1997), aunque Campbell y Hausenblas (2009) encuentran mayores efectos en poblaciones mayores en comparación con las poblaciones más jóvenes, pues la imagen negativa del cuerpo disminuye con la edad (Reboussin et al., 2000).

A principios del siglo XXI, empiezan a aflorar en España las prácticas deportivas de wellness y fitness (García Ferrando, 2006b), que orientan a las personas hacia el ejercicio físico con fines de salud y ofrecen al cliente una amplia y atractiva oferta de actividades, incluyendo variabilidad de servicios, modalidades adaptables a distintos grupos de población, disponibilidad horaria y actualización de servicios en función de la demanda (Sicilia, Águila, Orta, y Muyor, 2008). García Ferrando (2006a) encuentra que un $14 \%$ de los españoles practican gimnasia de mantenimiento, un $11,7 \%$ practican actividades como aeróbic, gym jazz o step, y un 6,8\% practican musculación en salas de gimnasio.

El medio en el que se desarrollan este tipo de actividades se asocian con una mayor preocupación por la imagen corporal para las mujeres, debido a que existe presencia masculina (Prichard y Tiggemann, 2005) y el cuerpo es el eje central (Greenleaf, McGreer, y Parham, 2006), exhibiéndose mediante el uso de ropa ajustada en una habitación diseñada para proporcionar información (Katula, McAuley, Mihalko, y Bane, 1998), provocando una excesiva comparación social y que se conciba el cuerpo como un objeto, y se incide extremadamente en la pérdida de peso de los usuarios (Prichard y Tiggemann, 2005). Estos mismos autores en 2008, explican que en particular, las mujeres que frecuentan estos centros se encuentran rodeadas de espejos y carteles que muestran el ideal del cuerpo, fomentando también la comparación social y una conciencia corporal elevada (Frederick y Shaw, 1995), pues ha sido demostrado que el uso de espejos produce sensaciones negativas en mujeres sedentarias, provocando insatisfacción con su imagen corporal (Martin Ginis, Jung, y Gauvin, 2003). En nuestra opinión, este hecho afecta también, aunque en menor grado, a la población masculina. 
En cuanto a los instructores de los centros fitness, Prichard y Tiggemann (2005) aseguran que tienen menos insatisfacción corporal que los usuarios, argumentando que es posible que sólo las personas que se sienten cómodas con su cuerpo están dispuestas a mostrarlo frente a tanta gente o que están habituadas al ambiente de estos centros y dejan de influir en la su percepción de la imagen corporal.

Pero la imagen que proporcionan los instructores influye en la percepción que los usuarios tienen de su propia imagen corporal. Así, Martin Ginis, Prapavessis y Haase (2008) descubrieron que cuanto mayor es la discrepancia percibida entre los participantes en actividades dirigidas y el instructor, mayor es la insatisfacción corporal después del ejercicio, sin embargo, Evans, Cotter y Roy (2005) muestran que las mujeres prefieren un instructor más delgado que los hombres, pero ambos géneros coinciden en que las características más importantes que éstos deben presentar son: buena forma física y entusiasmo.

No obstante, las clases de fitness se perciben como una ocasión social agradable para muchas mujeres (Maguire y Mansfield, 1998) y la investigación ha demostrado que, en general, las mujeres prefieren practicar ejercicio en grupos en lugar de solas (Burke, Carron, y Eys, 2006). Sin embargo, Focht y Hausenblas (2006) muestran que las mujeres con niveles elevados de ansiedad física social experimentan cambios afectivos negativos si realizan ejercicio en lugares públicos, y positivos si lo hacen en lugares privados.

En cuanto a los hombres, los que presentan altos niveles de ansiedad y vergüenza dejan de participar en actividades recreativas y sociales, y dedican el tiempo a entrenar en salas de musculación para acercarse a su imagen corporal ideal (Pope, Gruber, Choi, Olivardia, y Phillips, 1997).

Prichard y Tiggemann (2008) afirman que la gestión de los centros de fitness debe tener como objetivo proporcionar entornos positivos para la imagen corporal de sus usuarios, como por ejemplo, reducir el número de espejos en las salas, eliminar los carteles que muestren un cuerpo ideal y poco realista y contratar instructores saludables que no sean excesivamente delgados, que se centren en la promoción del disfrute y la salud por encima de motivos como la pérdida de peso, y que lleven y fomenten llevar ropa holgada (Fredrickson y Roberts, 1997).

\section{Instrumentos de ayuda}

A continuación presentamos una presentación de los instrumentos que pueden ser usados para conocer la imagen corporal que tienen los usuarios de los centros fitness.

Se emplearán diferentes escalas surgidas de los principales variables que explican se relacionan con el estudio de la imagen corporal:

- Contour Drawing Rating Scale (CDRS) diseñado por Thompson y Gray (1995). Se trata de una escala gráfica autoadministrable de siluetas femeninas para las mujeres, y masculinas para los hombres, que aumentan de tamaño a medida que aumenta la puntuación (de 1 a 9), cubriendo así un continuo de peso corporal que va desde figuras muy delgadas (puntuación 1 y 2), delgadas (puntuación 3 y 4), a figuras gruesas (puntuación 6 y 7) y muy gruesas (puntuación 8 y 9), pasando por 
una figura normal (puntuación 5). La discrepancia entre la figura actual e ideal es indicativa de distorsión perceptual.

- Body Image Avoidance Questionnaire (BIAQ). Diseñado por Rosen, Srebnik, Saltzberg y Wendt (1991). Está compuesto por 19 ítems que evalúan la frecuencia con la que uno se involucra en conductas de evitación relacionadas con la imagen corporal. Se compone de las siguientes subescalas: modo de llevar la ropa, restricción de la ingesta, actividades sociales y arreglarse y pesarse.

- Escala de Ansiedad Social de Física (SPAS) es un inventario de autoinforme de 12 ítems que fue diseñado por Hart et al. (1989) para evaluar el rasgo de ansiedad física social, es decir, la ansiedad asociada a la preocupación de que el propio cuerpo puede ser evaluado negativamente por otros. La escala tiene dos sub-escalas: sensación de malestar y la expectativa de la evaluación negativa. Los participantes respondieron a una escala de 5 puntos tipo Likert con las anclas de la nada (1), poco (2), moderada (3), muy (4), y muy (5). Las puntuaciones van de 12 a 60, con valores más altos indican una mayor ansiedad.

- Cuestionario de Auto-objetivación. Fue creado por Noll y Fredrickson (1998), que clasificar 10 atributos diferentes en orden descendente de importancia. Cinco de los 10 fueron los atributos relacionados con la apariencia (músculos definidos, apariencia sexual, el peso, atractivo físico, y las medidas del cuerpo), y los otros cinco se basaban en la competencia física (salud, fuerza, coordinación física, condición física, y nivel de energía). Puntuaciones posibles van desde -25 hasta 25, las puntuaciones más altas y positivas indican un mayor énfasis en la apariencia, que se interpreta como rasgo de alta auto-objetivación.

- Cuestionario de preferencia de vestimenta. (Prichard y Tiggerman, 2005). Preguntas acerca de lo importante que es para los sujetos llevar ropa de moda mientras practican ejercicio en el centro deportivo, empleando una escala de Likert de 5 puntos, desde 1 (nada importante) al 5 (muy importante). También pedir que indiquen en una escala del 1 (nunca) a 6 (siempre) la frecuencia con la que llevan ropa que cubren una gama de niveles de opresión cuando practican frecuentan el centro.

- Escala de Medida de los Motivos para la Actividad Física-Revisada en españoles (MPAM-R) de Ryan, Frederick, Lepes, Rubio, y Sheldon (1997) validada al español por Moreno, Cervelló y Martínez Camacho (2007). Esta escala mide los motivos de práctica hacia la actividad física a través de 30 ítems, agrupados en cinco factores: disfrute (p.g. "realizo actividad física porque es divertido"), apariencia (p. g. "realizo actividad física porque quiero mantener mi peso para tener buena imagen"), social (p.g. "realizo actividad física porque quiero conocer a nuevas personas"), fitness/salud (p.g. "realizo actividad física porque quiero mejorar mi aptitud cardiovascular") y competencia (p.g. "realizo actividad física porque me gustan los desafíos"). De las cinco subescalas, disfrute y competencia han sido utilizadas para reflejar una orientación motivacional intrínseca, mientras que las otras tres subescalas (social, fitness/salud y apariencia) reflejan varios niveles de motivación extrínseca. El sujeto responde a la cuestión "Realizo actividad física..." a través de los ítems que conforman la escala. Se mide a través de una escala tipo Likert de siete puntos donde 1 corresponde a "nada verdadero para mí" y 7 a "totalmente verdadero para mí". 


\section{REFERENCIAS}

Ackard, D. M., Brehm, B. J., y Steffen, J. J. (2002). Exercise and eating disorders in college-aged women: Profiling excessive exercisers. Eating Disorders, 10, 31-47.

Ahmed, C., Hilton, W., y Pituch, K. (2002). Relations of strength training to body image among a sample of female university students. Journal of Strength and Conditioning Research, 16, 645-648.

Allgood-Merten, B., Lewinsohn, P., y Hops, H. (1990). Sex differences and adolescent depression. Journal of Abnormal Psychology, 99, 55-63.

American Psychological Association (1994). Diagnostic and statistical manual of mental disorders (4ํㅡㄹición). Washington, DC: Author.

Asçı, H. F., Tüzün, M., y Koca, C. (2006). An examination of eating attitudes and physical activity levels of Turkish university students with regard to self-presentational concern. Eating Behaviors, 7, 362-368.

Atalay, A. A. y Gençöz, T. (2008). Critical Factors of Social Physique Anxiety: Exercising and Body Image Satisfaction. Behaviour Change, 25, 178-188.

Bas, M., Asçı, F.H., Karabudak, E., y Kiziltan, G. (2004). Eating attitudes and their psychological correlates among Turkish adolescents. Adolescence, 39, 593-599.

Burke, S. M., Carron, A. V. y Eys, M.A. (2006). Physical activity context: Preferences of university students. Psychology of Sport and Exercise, 7, 1-13.

Calogero, R. M., y Pedrotty, K. N. (2007). Daily practices for mindfull exercise. En L'Abate, L., Embry, D. y Baggett, M. (Eds.). Low-cost approaches to promote physical and mental health: Theory, research, and practice (pp.141-160). Arlington: Springer.

Campbell, A., y Hausenblas, A. (2009). Effects of Exercise Interventions on Body Image: A Meta-analysis. Journal of Health Psicology, 14, 780 - 793.

Cash, T. F. (1989). Body-image affect: Gestalt versus summing the parts. Perceptual and Motor Skills, 69, 17-18.

Cash, T. F. (1995). What do you see when you look in the mirror? Helping yourself to a positive body image. Nueva York: Bantam Books.

Cash, T. F. (2000). The Multidimensional Body-Self Relations Questionnaire users' manual. Disponible en: www.body-images.com (Consultado el 1-03-2011).

Daubenmier, J.J. (2005). The relationship of yoga, body awareness, and body responsiveness to self-objectification and disordered eating. Psychology of Women Quarterly, 29, 207-219.

Davis, C, Kennedy, S.H., Ravelski, E. y Dionne, M. (1994). The role of physical activity in the development and maintenance of eating disorders. Psychological Medicine. 24, 957-967.

Davis, C. y Cowles, M. (1991). Body Image and Exercise: A study of relationships and comparisons between physically active men and women. Sex Roles, 25, 33-45

Duncan, M. J., Al-Nakeeb, Y., Nevill, A., y Jones, M. V. (2004). Body image and physical activity in British secondary school children. European Physical Education Review, 10, 243-260.

Eagly, A. H., Ashmore, R. D., Makhijani, M. G., y Longo, L. C. (1991). What is beautiful is good but... A meta-analytic review of research on the physical attractiveness stereotype. Psychological Bulletin, 110, 109-128. 
Eklund, R. C., y Crawford, S. (1994). Active women, social physique anxiety, and exercise. Journal of Sport and Exercise Psychology, 16, 431-438.

Elgin, J. y Pritchard, M. (2006). Gender differences in disordered eating and its correlates. Eating and Weight Disorders, 11, 96-101.

Evans, R. R., Cotter, E. M. y Roy, J. L. P. (2005). Preferred body type of fitness instructors among university students in exercise classes. Perceptual and motor skills, 101(1), 257-266.

Feingold, A., y Mazzella, R. (1998). Gender differences in body image are increasing. Psychological Sciences, 9, 190-195.

Festinger, L. (1954). A theory of social comparison processes. Human Relations, 7, 117140.

Focht, B. C., y Hausenblas, H. A. (2006). Exercising in public and private environments: Effects on feeling states in women with social physique anxiety. Journal of Applied Biobehavioral Research, 11, 147-165.

Franzoi, S.L. (1995). The body-as-object versus the body-as-process: Gender differences and gender considerations. Sex Roles, 33, 417-437.

Franzoi, S.L. y Klaiber, J.R. (2007). Body use and reference group impact: With whom do we compare our bodies? Sex Roles, 56, 205-214.

Frederick, C. y Shaw, S. (1995). Body image as a leisure constraint: Examining the experience of aerobic exercise classes for young women. Leisure Sciences, 17, 57-73.

Fredrickson, B. L., y Roberts, T. (1997). Objectification theory: Toward understanding women's lived experiences and mental health risks. Psychology of Women Quarterly, 21, 173-206.

García Ferrando, M. (2006a), Posmodernidad y Deporte: Entre la Individualiuzación y la Masificación. Encuesta hábitos deportivos de los españoles 2005. Madrid: CIS/Siglo XXI.

García Ferrando, M. (2006b). Veinticinco años de análisis del comportamiento deportivo de la población española (1980-2005). Revista internacional de sociología, 64 (44), 15-38.

Garner, D. M., Garfinkel, P. R., Schwartz, D., y Thompson, M. (1980). Cultural expectations of thinness in women. Psychological Reports, 47, 483-491.

Garner, D.M. (1997). The body image survey. Psychology Today, 31, 32-84.

Garner, D.M. (2002). Body image and anorexia nervosa. En Cash, T. F. y Pruzinsky, T. (Eds.). Body image: A handbook of theory, research, and clinical practice (pp. 295303). Nueva York: Guilford.

Greenleaf, C., McGreer, R., y Parham, H. (2006). Physique attitudes and self-presentation concerns: Exploratory interviews with female group aerobic exercisers and instructors. Sex Roles, 55, 189-199.

Harrison, K. y Cantor, J. (1997). The relationship between media consumption and eating disorders. Journal of Communication, 7, 40-61.

Hart, E. A., Leary, M. R., y Rejeski, W. J. (1989). The measurement of social physique anxiety. Journal of Sport and Exercise Psychology, 11, 94-104.

Hausenblas, H.A. y Fallon, E.A. (2002). Relationship among Body Image, Exercise Behavior, and Exercise Dependence Symptoms. International Journal of Eating Disorders, 32(2), 179-185. 
Hausenblas, H. A., y Fallon, E. A. (2006). Exercise and body image: A meta-analysis. Psychology and Health, 21(1), 33-47.

Heinberg, L. J. (1995). Theories of body image disturbance. En Thompson, J.K. (Ed.). Body image, eating disorders and obesity: An integrative guide for assessment and treatment. Washington, D. C.: American Psychological Association.

Henry, R. N., Anshel, M. H., y Michael, T. (2006). Effects of Aerobic and Circuit Training on Fitness and Body Image among Women. Journal of sport Behavior, 29, 281303.

Johnson, A., Steinberg, R., y Lewis, W. (1988). Bulimia. En Clark, K., Parr, R. y Castelli, W. (Eds.). Evaluation and management of eating disorders, anorexia, bulimia and obesity (pp. 177-190). Illinois: Life Enhancement Publications.

Katula, J. A., McAuley, E., Mihalko, S. L., y Bane, S. M. (1998). Mirror, mirror on the wall Exercise environment influences on self-efficacy. Journal of Social Behavior and Personality, 13, 319-332.

Koff, E., y Bauman, C. L. (1997). Effects of wellness, fitness, and sport skills programs on body image and lifestyle behaviors. Perceptual and Motor Skills, 84, 555-562.

Koff, E., Rierdan, J., y Stubbs, M. L. (1990). Gender, body image, and self-concept in early adolescence. Journal of Early Adolescence, 10, 56-68.

Koyuncu, M., Serdar, T., Canpolat, A. M., y Catikkas, F. (2010). Body Image Satisfaction and dissatisfaction, Social Physique Anxiety, Self-esteem, And Body Fat Ratio in female exercisers and nonexercisers. Social behabvior and personality, 38(4), 561-570.

Krane, V., Stiles-Shipley, J.A., Waldron, J., y Michalenok, J. (2001). Relationships among body satisfaction, social physique anxiety, and eating behaviors in female athletes and exercisers. Journal of Sport Behavior, 24, 247-264.

Lantz, C. D., Hardy, C. J., y Ainsworth, B. E. (1997). Social physique anxiety and perceived exercise behavior. Journal of Sport Behavior, 20, 83-93.

Leary, M. R. (1992). Self-presentational processes in exercise and sport. Journal of Sport and Exercise Psychology, 14, 339-351.

LePage, M. L., y Crowther, J. H. (2010). The effects of exercise on body satisfaction and affect. Body image, 7, 124-130.

Maguire, J., y Mansfield, L. (1998). No-body's perfect: Women, aerobics, and the body beautiful. Sociology of Sport Journal, 15, 109-137.

Maltby, J., y Day, L. (2001). The relationship between exercise motives and psychological well-being. The Journal of Psychology, 135, 651-660.

Martin, K. A., Jung, M. E., y Gauvin, L. (2003). To see or not to see: Effects of exercising in mirrored environments on sedentary women's feeling states and self-efficacy. Health Psychology, 22, 354-361.

Martin, K. A., Prapavessis, H., y Haase, A. M. (2008). The effects of physique-salient and physique non-salient exercise videos on women's body image, self-presentational concerns, and exercise motivation. Body Image, 5, 164-172.

Martin, J. J., Engels, H. J., Wirth, J. C. y Smith, K. L. (1997). Predictors of social physique anxiety in elite female youth athletes. Women in Sport and Physical Activity Journal, 6, 29-48.

McCreary, D. R., Hildebrandt, T. B., Heinberg, L. J., Boroughs, M., y Thompson, J. K. (2007). A Review of Body Image Influences on Men's Fitness Goals and Supplement Use. American Journal of Men's Health, 1(4), 307-316. 
Mond, J. M., Hay, P. J., Rodgers, B., y Owen, C. (2006). An update on the definition of "excessive exercise" in eating disorder research. International Journal of Eating Disorders, 39, 147-153.

Moreno, J. A., Cervelló, E., y Martínez Camacho, A. (2007b). Validación de la Escala de Medida de los Motivos para la Actividad Física-Revisada en españoles: Diferencias por motivos de participación. Anales de Psicología, 23, 167-176.

Noll, S. M., y Frederickson, B. L. (1998). A mediational model linking self- objectification, bodyshame, and disordered eating. Psychology of Women Quarterly, 22, 623-636.

Pope, H. G., Gruber, A. J., Choi, P., Olivardia, R. y Phillips, K. A. (1997). Muscle dysmorphia: An underrecognized form of body dysmorphic disorder. Psychosomatics, 38, 548-557.

Prichard, I., y Tiggemann, M. (2005). Objectification in fitness centers: Self-objectification, body dissatisfaction, and disordered eating in aerobic instructors and aerobic participants. Sex Roles, 53, 19-28.

Prichard, I., y Tiggeman, M. (2008). Relations among exercise type, self-objectification, and body image in the fitness center environment: The role of reasons for exercise. Psychology of Sport and Exercise, 9, 855-866.

Reboussin, B. A., Rejeski, W. J., Martin, K. A., Callahan, K., Dunn, A. L., King, A. C., et al. (2000). Correlates of satisfaction with body function and body appearance in middle- and older aged adults: The activity counseling trial (ACT). Psychology and Health, 15, 239-254.

Rosen, J. C., Srebnik, D., Saltzberg, E. y Wendt, S. (1991). Development of a body image avoidance questionnaire. Journal of Consulting and clinical Psychology, 3, 32-37.

Rowe, D. A. (1999). Development of the body self-image questionnaire. Measurement in Physical Education and Exercise Science, 3, 223-247.

Russell, W. D. (2002). Comparison of self-esteem, body satisfaction, and social physique anxiety across males of different exercise frequency and racial background. Journal of Sport Behavior, 25, 74-90.

Ryan, R. M., Frederick, C. M., Lepes, D., Rubio, N., y Sheldon, K. M. (1997). Intrinsic motivation and exercise adherence. International Journal of Sport Psychology, 28, 335-354.

Sabiston, C. M., Crocker, P. R. E., y Munroe-Chandler, K. J. (2005). Examining currentideal discrepancy scores and exercise motivations as predictors of social physique anxiety in exercising females. Journal of Sport Behavior, 28, 68-85.

Salovey, P., y Rodin, J. (1984). Some antecedents and consequences of socialcomparison jealousy. Journal of Personality and Social Psychology, 47, 780-792.

Sicilia, A., Águila, C., Orta, A., y Muyor, J. M. (2008). Perfil de usuarios deportivos. Almería: Universidad de Almería.

Skrinar, G. S., Bullen, B. A., Cheek, J., McArthur, J. W., y Vaughan, L. K. (1986). Effects of endurance training on body-consciousness in women. Perceptual and Motor Skills, 62, 483-490.

Strelan, P., Mehaffey, S., y Tiggemann, M. (2003). Self-objectification and esterrm in young women: the mediating role of reasons for exercise. Sex Roles: A Journal of Research, 48, 89-95.

Thompson, J. K., Heinberg, L. J., Altabe, M., y Tantleff-Dunn, S. (1999). Exacting beauty: Theory, assessment, and treatment of body image disturbance. Washington, D. C.: American Psychological Association. 
Thompson, M. A., y Gray, J. J. (1995). Development and validation of a new body-image assessment scale. Journal of Personal Assessment, 64, 258-69.

Tiggemann, M., y Williamson, S. (2000). The effect of exercise on body satisfaction and self-esteem as a function of gender and age. Sex Roles, 44, 119-129.

Tucker, L. A., y Maxwell, K. (1992). Effects of weight-training on the emotional well-being and body image of females: Predictors of greatest benefit. American Journal of Health Promotion, 6, 338-344.

Tucker, L. A., y Mortell, R. (1994). Comparison of the effects of walking and weighttraining program on body image in middle-aged women: An experimental study. American Journal of Health Promotion, 8, 34-42.

Van den Buick, J. (2000). Is television bad for your health? Behavior and body image of the adolescent "couch potato." Journal of Youth and Adolescence, 29, 273-281.

Wasylkiw, L., Emms, A. A., Meuse, R. y Poirier, K. F. (2009). Are all models created equal? A content analysis of advertisements of fitness versus fashion magazines. Body Image, 6, 137.140.

Williams, P. A. y Cash, T. F. (2001). Effects of a circuit weight-training program on the body images of college students. The International Journal of Eating Disorders, 30, 75-82. 